Discrete Comput Geom 28:151-174 (2002)

DOI: $10.1007 / \mathrm{s} 00454-002-2757-9$

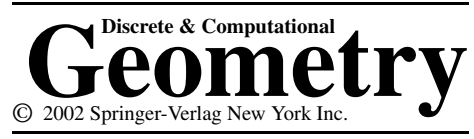

\title{
Combinatorics and Quotients of Toric Varieties
}

\author{
Yi Hu \\ Department of Mathematics, University of Arizona, \\ Tucson, AZ 85721, USA \\ yhu@math.arizona.edu
}

\begin{abstract}
For linear projections of polytopes and fans of cones we introduce some new objects such as: virtual chambers, virtual cones and (locally) coherent costrings. Virtual chambers (cones) generalize real chambers (cones), while (locally) coherent costrings are linear dual to (locally) coherent strings. We establish various correspondences for these objects and their connections to toric geometry.
\end{abstract}

\section{Introduction}

This paper studies two related subjects. One is some combinatorics arising from linear projections of polytopes and fans of cones. The other is quotient varieties of toric varieties. The relation is that projections of polytopes are related to quotients of projective toric varieties and projection of fans are related to quotients of general toric varieties. Despite its relation to geometry the first part is purely combinatorial and should be of interest in its own right.

For the combinatorial part, consider a surjective linear map $\pi: V \rightarrow W$ between two real vector spaces. Let $P$ be a polytope in $V$ of full dimension and let $Q=\pi(P)$. The projections of all faces of $P$ under $\pi$ induce a polytopal subdivision of $Q$. This gives rise to the poset $\Gamma=\Gamma(P, Q)$ of the so-called chambers in $Q$ (Definition 1.1).

The sequence $0 \rightarrow \operatorname{ker} \pi \rightarrow V \stackrel{\pi}{\rightarrow} W \rightarrow 0$ induces the dual sequence

$$
0 \rightarrow W^{*} \rightarrow V^{*} \stackrel{\pi^{\vee}}{\longrightarrow}(\operatorname{ker} \pi)^{*} \rightarrow 0
$$

The projections of all cones of the normal fan $\Delta(P)$ of $P$ under $\pi^{\vee}$ induce a fan in $(\operatorname{ker} \pi)^{*}$. This is the normal fan $\Delta(\Sigma(P, Q))$ of the Billera-Sturmfels fiber polytope $\Sigma(P, Q)$. Let $\Gamma^{*}=\Gamma^{*}(P, Q)$ be the poset of cones in this fan (Definition 1.2). 
The above descriptions clearly put $\Gamma$ and $\Gamma^{*}$ in a linear dual situation. Indeed, they are part of a larger picture which goes beyond just dual analogy.

First, it has been known that $\Gamma^{*}$ is in one-to-one correspondence with the poset $\mathcal{T}_{\text {coh }}=\mathcal{T}_{\text {coh }}(P, Q)$ of the so-called coherent strings of the projection $\pi: P \rightarrow Q$ (see Definition 2.1). The poset $\mathcal{T}=\mathcal{T}(P, Q)$ of locally coherent strings, the generalizations of coherent strings, has also been around for quite a while (see Definition 3.1). Investigating this, it is quite natural to ask the following questions:

(1) What are the generalizations of elements in $\Gamma^{*}$ so that they correspond to locally coherent strings in $\mathcal{T}$ ?

(2) Are there coherent costrings linear dual to coherent strings?

(3) Are there locally coherent costrings linear dual to locally coherent strings?

(4) Are there perfect correspondences for these new objects similar to the correspondence between $\Gamma^{*}$ and $\mathcal{T}_{\text {coh }}$ ?

Indeed, perfect correspondences do occur and are the main theme of this paper. To this end, some new objects are to be introduced in response to each and every one of the above questions:

(1) The poset $\Gamma_{\mathrm{vir}}^{*}=\Gamma_{\mathrm{vir}}^{*}(P, Q)$ of virtual cones of the projection $\pi^{\vee}$. A virtual cone is a combinatorial abstraction that generalizes a real polyhedral cone (Definition 7.1). It corresponds to a locally coherent string of $\pi: P \rightarrow Q$.

(2) The poset $\mathcal{T}_{\text {coh }}^{*}=\mathcal{T}_{\text {coh }}^{*}(P, Q)$ of coherent costrings of the projection $\pi^{\vee}$. This notion is linear dual to that of coherent strings. A coherent costring is roughly speaking a special lifting of a fan via the map $\pi^{\vee}$ (Definition 4.2). It corresponds to a chamber in $\Gamma$.

(3) The poset $\mathcal{T}^{*}=\mathcal{T}^{*}(P, Q)$ of locally coherent costrings of the projection $\pi^{\vee}$. This notion is linear dual to that of locally coherent strings. A locally coherent costring is roughly speaking a lifting of a fan via the map $\pi^{\vee}$ (Definition 5.1).

(4) The poset $\Gamma_{\text {vir }}=\Gamma_{\text {vir }}(P, Q)$ of virtual chambers (cells) of the projection $\pi$. A virtual chamber (cell) is a combinatorial abstraction that generalizes a real chamber (cell) in $\Gamma$ (Definition 6.1). It corresponds to a locally coherent costring.

Locally coherent strings first appeared in [7] when $\operatorname{dim} Q=1$ and were generalized and studied afterwards, especially in association with the generalized Baues conjecture (see [30] for a recent survey). Virtual chambers for a simplex were introduced and studied in [13].

Our first main result in this paper is

Theorem A (Theorem 7.3). Let the notations be as explained in the above. Then:

(1) $\mathcal{T}(P, Q)$ is canonically anti-isomorphic to $\Gamma_{\text {vir }}^{*}(P, Q)$. The isomorphism extends the anti-isomorphism between $\mathcal{T}_{\mathrm{coh}}(P, Q)$ and $\Gamma^{*}(P, Q)$.

(2) $\mathcal{T}^{*}(P, Q)$ is canonically anti-isomorphic to $\Gamma_{\mathrm{vir}}(P, Q)$. The isomorphism extends the anti-isomorphism between $\mathcal{T}_{\text {coh }}^{*}(P, Q)$ and $\Gamma(P, Q)$.

As hinted in the beginning, the above combinatorial objects underlie some quotient toric geometry. We now elaborate. 
Let $X_{\Delta_{0}}=\bigcup_{\sigma \in \Delta_{0}} A_{\sigma}$ be the toric variety over the complex number field defined by an arbitrary fan $\Delta_{0}$ with a lattice $N$, where $A_{\sigma}$ is the affine variety defined by the cone $\sigma$ in $\Delta_{0}$. Let $M^{2}$ be any sublattice of the dual lattice $M$ of $N$. Consider the exact sequence

$$
0 \rightarrow M^{2} \rightarrow M \stackrel{\pi}{\rightarrow} M^{1} \rightarrow 0
$$

where $M^{1}=M / M^{2}$. Let

$$
0 \rightarrow N^{1} \rightarrow N \stackrel{\pi^{\vee}}{\longrightarrow} N^{2}
$$

be the dual exact sequence where the image of $\pi^{\vee}$ has finite index in $N^{2}$. If we apply $\operatorname{Hom}_{\mathbb{Z}}\left(-, \mathbb{C}^{*}\right)$ to the original sequence, then we get the exact sequence

$$
1 \rightarrow \operatorname{Hom}_{\mathbb{Z}}\left(M^{1}, \mathbb{C}^{*}\right) \rightarrow \operatorname{Hom}_{\mathbb{Z}}\left(M, \mathbb{C}^{*}\right) \rightarrow \operatorname{Hom}_{\mathbb{Z}}\left(M^{2}, \mathbb{C}^{*}\right) \rightarrow 1 .
$$

The group $G=\operatorname{Hom}_{\mathbb{Z}}\left(M^{1}, \mathbb{C}^{*}\right)$ is a subgroup of the torus $\operatorname{Hom}_{\mathbb{Z}}\left(M, \mathbb{C}^{*}\right)$ and is the product of the torus $\operatorname{Hom}_{\mathbb{Z}}\left(M_{\text {free }}^{1}, \mathbb{C}^{*}\right)$ and the finite group $\operatorname{Hom}_{\mathbb{Z}}\left(M_{\mathrm{tor}}^{1}, \mathbb{C}^{*}\right)$. This is a diagonalizable subgroup but not a subtorus in general.

The projective quotients of projective toric varieties by subtori have been studied by Kapranov et al. in [21]. In short, [21] shows that the real chambers (cells) correspond to projective toric quotients whose fans are those induced by the corresponding global coherent costrings. Theorem $\mathrm{B}$ and Corollary $\mathrm{C}$ below naturally extend their results to the quotients of $X_{\Delta_{0}}$ by the diagonalizable group $G$ and exhibit the underlying geometry behind the combinatorial bijection described in Theorem $\mathrm{A}(2)$.

Theorem $\mathbf{B}$ (Theorem 10.5). Let $\Delta \subset \Delta_{0}$ be a subset of cones and let $U=\bigcup_{\sigma \in \Delta} A_{\sigma}$ be an open subset of $X_{\Delta_{0}}$ where $A_{\sigma}$ is the affine open subset defined by $\sigma$. Then:

(1) $U$ has a categorical quotient by the action of $G$ if $\Delta$ is a locally coherent costring of the projection $\pi^{\vee}$ and every cone in $\pi^{\vee}(\Delta)$ is strongly convex. In this case the quotient $U / / G$ is isomorphic to $X_{\pi^{\vee}(\Delta)}$, the toric variety defined by the induced fan $\pi^{\vee}(\Delta)$.

(2) $U / / G$ is geometric if and only if $\operatorname{dim} \pi^{\vee}(\sigma)=\operatorname{dim} \sigma$ for all $\sigma \in \Delta$ (i.e., $\Delta$ is tight by definition, or, equivalently, minimal in $\mathcal{T}^{*}$ ).

When $X_{\Delta_{0}}$ is projective and equipped with a $T$-linearized ample line bundle $L$, it is associated to a polytope $P$ in the lattice $M$. Let $Q=\pi(P)$. Then by the equivalence of (2) in Theorem A, we have

Corollary C. Every virtual chamber (cell) in $\Gamma_{\mathrm{vir}}(P, Q)$ defines a (not necessarily projective) toric quotient variety of $X_{\Delta_{0}}$ whose fan is induced from the corresponding locally coherent costring in $\mathcal{T}^{*}(P, Q)$.

At the end of this paper we try to relate the combinatorial bistellar flips to the geometric Mori-type flips among quotients of affine spaces.

We point out here that there are some recent works that study the quotients of toric varieties by subtori [5], [6], [1], [2]. In particular, as pointed out by one of the 
referees, Hamm [18] and Świecicka [32] both independently gave a characterization of all invariant open subsets of a toric variety having a good quotient with respect to a given subtorus. Hence their results may be translated into the terminology of a locally coherent costring. Our approach is different from theirs. This paper deals with the fruitful interaction between polyhedral combinatorics and toric algebraic geometry. Separating the geometric part from the combinatorial part will undermine this beautiful connection. For example, Theorem B and Corollary C are direct results of Theorem $\mathrm{A}(2)$ and exhibit the underlying geometry behind it. This establishes (for the first time, I hope) that a virtual chamber corresponds to a quotient toric variety whose fan is given by the corresponding locally coherent costring and this naturally extends the old, well-known bijection between genuine chambers (projective quotients) and coherent costrings (projective fans). Another minor difference here is that the group $G$ that we use to take the quotient arises naturally as a diagonalizable subgroup but not a subtorus in general (this agrees with Cox's quotient construction of a toric variety). Lastly, our unfulfilled ambition on the combinatorial part substantially delayed the publication of our geometric part.

Finally I (first) learned from V. Reiner that Santos [31] has obtained some important results on Baues conjectures which are related to our combinatorial constructions.

The paper is structured as follows. From Sections 1 to 3, we collect some basic results that we will need. Our main source (not necessarily original) is [33]. In Sections 4-7 we introduce our various new combinatorial concepts and prove their basic properties. In Section 8 we study "realizable virtual cells" and "lexicographic cells". The rest of the paper is devoted to exploring the toric geometry underlying our combinatorial theory. Our main references here are [16], [12], [21] and [7].

\section{Chambers and Cones}

Let $V$ and $W$ be vector spaces over $\mathbb{R}$ of finite dimensions, and let

$$
\pi: V \rightarrow W
$$

be a linear projection. Assume that $P \subset V$ is a polytope of full dimension and $Q=\pi(P)$.

Definition 1.1. The (polytopal) cell in $Q$ containing a point $q \in Q$ is the intersection of $\pi$-images of all the faces of $P$ that contain the given point $q$, that is,

$$
\mathbf{c}(q):=[q]:=\bigcap\{\pi(F) \mid q \in \pi(F), F \in L(P)\} .
$$

The set of all cells is denoted by $\Gamma=\Gamma(P, Q)$, partially ordered by inclusion. Maximal cells are called chambers. ${ }^{1}$

\footnotetext{
${ }^{1}$ Cells are often called chambers in other literature. Then chambers in our sense would have to be called maximal chambers.
} 
Dually, the sequence $0 \rightarrow \operatorname{ker} \pi \rightarrow V \stackrel{\pi}{\rightarrow} W \rightarrow 0$ induces the sequence

$$
0 \rightarrow W^{*} \rightarrow V^{*} \stackrel{\pi^{\vee}}{\longrightarrow}(\operatorname{ker} \pi)^{*} \rightarrow 0 .
$$

Recall that given any linear function $\psi \in V^{*}$, the set $P^{\psi}$ of all points in $P$ on which $\psi$ is maximal is a face of $P$, and all non-empty faces of $P$ arise in this way. In fact, given any $F$ in the face lattice $L(P)$ of $P$, the set of $\psi \in V^{*}$ such that $P^{\psi}=F$ is a cone, called the normal cone of $F$, and is denoted by $N(P, F)=N(F)$. The set $\Delta(P)$ of the normal cones of all faces of $P$ is the normal fan of $P$.

Definition 1.2. The (polytopal) cone in $(\operatorname{ker} \pi)^{*}$ containing a point $\psi \in(\operatorname{ker} \pi)^{*}$ is the intersection of all $\pi^{\vee}$-images of the cones in $\Delta(P)$ that contain $\psi$, that is,

$$
\sigma(\psi):=[\psi]:=\bigcap\left\{\pi^{\vee} N(P, F) \mid F \in L(P), \psi \in \pi^{\vee} N(P, F)\right\} .
$$

We use $\Gamma^{*}=\Gamma^{*}(P, Q)$ to denote the set of all those cones partially ordered by inclusion. (These cones form the normal fan of the fiber polytope $\Sigma(P, Q)[8])$.

Consider the polytope $P_{q}=\pi^{-1}(q)$ for any $q \in Q$. For any linear function $\psi \in$ $(\operatorname{ker} \pi)^{*}$, let $P_{q}^{\psi}$ be the non-empty face of $P_{q}$ defined by $\psi$. The normal cone $N\left(P_{q}, P_{q}^{\psi}\right)$ $=N\left(P_{q}^{\psi}\right)$ depends only on the cell $\mathbf{c}=\mathbf{c}(q)$. So, we may use $\Delta(\mathbf{c})$ to be the normal fan $\Delta\left(P_{q}\right)$ for all $q \in$ relint $\mathbf{c}$, where "relint" denotes "relative interior". For each $\psi$, let $[\psi]_{\mathbf{c}}$ denote the minimal cone in $\Delta(\mathbf{c})$ that contains $\psi$. Note that $\Delta(\mathbf{c})$ is a common refinement of $\Delta\left(\mathbf{c}^{\prime}\right)$ for all $\mathbf{c}^{\prime} \leq \mathbf{c}$.

In addition, for each face $P_{q}^{\psi}$ of $P_{q}$ there exists a unique minimal face of $P$ that contains $P_{q}^{\psi}$ : the intersection of all faces that contain $P_{q}^{\psi}$. We may use $F_{q, \psi}$ to denote this face of $P$. Since it only depends on the cell $\mathbf{c}=\mathbf{c}(\psi)$, we may write it as $F_{\mathbf{c}, \psi}$. Moreover, $F_{\mathbf{c}, \psi}$ only depends on the cone $[\psi]_{\mathbf{c}}$. So, we often denote it by $F_{\mathbf{c},[\psi] \mathbf{c}}$. Note that $F_{\mathbf{c}, \psi}=F_{\mathbf{c}, \psi^{\prime}}$ if and only if $[\psi]_{\mathbf{c}}=\left[\psi^{\prime}\right]_{\mathbf{c}}$. Note also that $N\left(P, F_{q, \psi}\right)$ projects onto $N\left(P_{q}, P_{q}^{\psi}\right)$ under the projection $\pi^{\vee}$.

Having the above explained, we can conclude that the fan of cones in Definition 1.2 is the common refinement of all the normal fans $\Delta(\mathbf{c})(\mathbf{c} \in \Gamma)$.

\section{Coherent Strings}

Given any linear function $\psi \in(\operatorname{ker} \pi)^{*}$, we have a collection of faces in $L(P)$ defined as follows:

$$
\mathcal{F}(\psi)=\left\{F_{\mathbf{c}, \psi} \mid \mathbf{c} \in \Gamma\right\} .
$$

(Throughout the paper the letter $\mathcal{F}$ denotes a collection of faces of a given polytope. For example, $\mathcal{F}(\psi)$ is the collection of faces defined by $\psi$.)

Definition 2.1 (see [33]). The above collection $\mathcal{F}(\psi)=\left\{F_{\mathbf{c}, \psi} \mid \mathbf{c} \in \Gamma\right\}$ of faces of $P$ is called a (global) coherent string of the projection $\pi: P \rightarrow Q$. We set $\mathcal{T}_{\text {coh }}=$ $\mathcal{T}_{\text {coh }}(P, Q)$ to be the set of all coherent strings of $\pi: P \rightarrow Q$, partially ordered by $\mathcal{F}(\psi) \leq \mathcal{F}\left(\psi^{\prime}\right)$ if $\bigcup \mathcal{F}(\psi) \subset \bigcup \mathcal{F}\left(\psi^{\prime}\right)$. 
Proposition 2.2. Given any linear function $\psi \in(\operatorname{ker} \pi)^{*}$, the coherent string $\mathcal{F}(\psi)$ satisfies the following properties:

(1) $\left\{\pi\left(F_{\mathbf{c}, \psi}\right) \mid \mathbf{c} \in \Gamma\right\}$ is a polytopal subdivision of $Q$ without repetitions;

(2) $\pi\left(F_{\mathbf{c}^{\prime}, \psi}\right) \subset \pi\left(F_{\mathbf{c}, \psi}\right)$ if and only if $F_{\mathbf{c}^{\prime}, \psi}=\pi^{-1}\left(\pi\left(F_{\mathbf{c}^{\prime}, \psi}\right)\right) \cap F_{\mathbf{c}, \psi}$.

Proof. Obviously, we have

$$
\bigcup\left\{\pi\left(F_{\mathbf{c}, \psi}\right) \mid \mathbf{c} \in \Gamma\right\}=\bigcup \mathbf{c}=Q .
$$

However, $q \in$ relint $\pi\left(F_{\mathbf{c}, \psi}\right) \cap$ relint $\pi\left(F_{\mathbf{c}^{\prime}, \psi}\right) \neq \emptyset$ if and only if $F_{\mathbf{c}, \psi}=\bigcap_{F \supset P_{q}^{\psi}} F=$ $F_{\mathbf{c}^{\prime}, \psi}$. This proves (1). (2) follows easily.

Lemma 2.3. $\mathcal{F}(\psi)=\mathcal{F}\left(\psi^{\prime}\right)$ if and only if $\sigma(\psi)=\sigma\left(\psi^{\prime}\right)$.

Proof. $\mathcal{F}(\psi)=\mathcal{F}\left(\psi^{\prime}\right)$ if and only if $F_{\mathbf{c}, \psi}=F_{\mathbf{c}, \psi^{\prime}}$ for all $\mathbf{c} \in \Gamma$ if and only if $[\psi]_{\mathbf{c}}=\left[\psi^{\prime}\right]_{\mathbf{c}}$ for all $\mathbf{c} \in \Gamma$ if and only if $\sigma(\psi)=\sigma\left(\psi^{\prime}\right)$.

So, we may denote a coherent string by $\mathcal{F}(\sigma)$ for some $\sigma \in \Gamma^{*}$. Given any point $q \in Q$, we also use $[q]_{\sigma}$ to denote $\pi\left(F_{q, \sigma}\right)$. Observe that $F_{q, \sigma}=F_{q^{\prime}, \sigma}$ if and only if $[q]_{\sigma}=\left[q^{\prime}\right]_{\sigma}$.

Remark 2.4. Notice that two different coherent strings may induce an identical subdivision of $Q$. This will not be the case if $P$ and $\pi(P)$ have the same numbers of vertices (thanks to a remark of one of the referees).

Proposition 2.5 [8]. The poset $\mathcal{T}_{\text {coh }}$ of all coherent strings and the poset $\Gamma^{*}$ are antiisomorphic. That is, $\mathcal{T}_{\text {coh }} \cong\left(\Gamma^{*}\right)^{\text {opp }}$ and $\Gamma^{*} \cong\left(\mathcal{T}_{\text {coh }}\right)^{\text {opp }}$.

Proof. Clearly the function $\mathcal{F}: \Gamma^{*} \rightarrow \mathcal{T}_{\text {coh }}$ is a bijection by the definition and Lemma 2.3. Let $\sigma$ be a cone and let $\mathcal{F}(\sigma)$ be its corresponding coherent string. Assume that $\sigma \leq \sigma^{\prime}$. Pick $\psi \in \sigma$ and $\psi^{\prime} \in \sigma^{\prime}$. Then $P_{q}^{\psi} \geq P_{q}^{\psi^{\prime}}$. That is, $F_{q, \psi} \geq F_{q, \psi^{\prime}}$. Since $\mathcal{F}(\sigma)=\left\{F_{q, \psi} \mid q \in Q\right\}$ and $\mathcal{F}\left(\sigma^{\prime}\right)=\left\{F_{q, \psi^{\prime}} \mid q \in Q\right\}$, we obtain that $\mathcal{F}(\sigma) \geq \mathcal{F}\left(\sigma^{\prime}\right)$. That is, the function $\mathcal{F}: \Gamma^{*} \rightarrow \mathcal{T}_{\text {coh }}$ is an order-reversing bijection.

\section{Locally Coherent Strings}

Locally coherent strings are a natural generalization of coherent strings. Geometrically, they are "continuous liftings of polytopal subdivisions of $Q$ " to the face lattice of $P$. It turns out that all of these things can be constructed as follows.

Consider an arbitrary map

$$
\tilde{\Psi}: \Gamma \rightarrow(\operatorname{ker} \pi)^{*} .
$$

It gives rise to a collection of faces of $P$ as follows:

$$
\mathcal{F}(\tilde{\Psi})=\left\{F_{\mathbf{c}, \tilde{\Psi}(\mathbf{c})} \mid \mathbf{c} \in \Gamma\right\}
$$


Definition 3.1 (see [33]). The collection $\mathcal{F}(\tilde{\Psi})$ of faces of $P$ is called a locally coherent string if $\tilde{\Psi}$ satisfies the so-called locally coherent condition: that is, for any $\mathbf{c} \geq \mathbf{c}^{\prime}$,

$$
\operatorname{relint}[\tilde{\Psi}(\mathbf{c})]_{\mathbf{c}} \subset \operatorname{relint}\left[\tilde{\Psi}\left(\mathbf{c}^{\prime}\right)\right]_{\mathbf{c}^{\prime}} .
$$

We set $\mathcal{T}=\mathcal{T}(P, Q)$ to denote the set of all locally coherent strings, partially ordered by $\tilde{\Psi} \leq \tilde{\Psi}^{\prime}$ if and only if $\bigcup \mathcal{F}(\tilde{\Psi}) \subset \bigcup \mathcal{F}\left(\tilde{\Psi}^{\prime}\right)$.

One can check that the locally coherent condition is equivalent to

$$
\text { relint } \pi^{\vee}\left(N\left(F_{\mathbf{c}, \tilde{\Psi}(\mathbf{c})}\right)\right) \subset \operatorname{relint} \pi^{\vee}\left(N\left(F_{\mathbf{c}^{\prime}, \tilde{\Psi}\left(\mathbf{c}^{\prime}\right)}\right)\right) .
$$

Proposition 3.2 [7], [28], [29]. Assume that a map $\tilde{\Psi}: \Gamma \rightarrow(\operatorname{ker} \pi)^{*}$ satisfies the locally coherent condition. Then the locally coherent string $\mathcal{F}(\tilde{\Psi})$ enjoys:

(1) $\{\pi(F) \mid F \in \mathcal{F}(\tilde{\Psi})\}$ is a subdivision of $Q$ without repetition.

(2) $\pi(F) \subset \pi\left(F^{\prime}\right)$ if and only if $F=F^{\prime} \cap \pi^{-1}(\pi(F))$ for any $F, F^{\prime} \in \mathcal{F}(\tilde{\Psi})$.

Furthermore, every collection of the faces of $P$ satisfying (1) and (2) arises in this way.

Proof. The theorem was proved in [7] for the case when $\operatorname{dim} Q=1$ and was explicitly pointed out in [29] for the general cases.

We adopt a proof suggested by one of the referees. The proof reduces the argument to the coherent case (Proposition 2.2) by using the following observation: for every pair $(\mathbf{c}, \sigma)$ of a cell $\mathbf{c} \in \Gamma$ and a cone $\sigma \in \Gamma^{*}$ there is precisely one face $F$ of $P$ such that the projection of the relative interior of $F$ contains the relative interior of $\mathbf{c}$ and the projection of the relative interior of $N(F)$ contains the relative interior of $\sigma$. We have $F=F_{\mathbf{c}, \psi}$ for any $\psi \in \sigma$.

Now if the map $\tilde{\Psi}$ satisfies the local coherence condition and $\mathbf{c}^{\prime}<\mathbf{c}$, then $F_{\mathbf{c}, \tilde{\Psi}(\mathbf{c})}=$ $F_{\mathbf{c}, \psi}$ and $F_{\mathbf{c}^{\prime}, \tilde{\Psi}\left(\mathbf{c}^{\prime}\right)}=F_{\mathbf{c}^{\prime}, \psi}$ for some $\psi$ in relint $[\tilde{\Psi}(\mathbf{c})]_{\mathbf{c}}$. So the claim follows from the coherent case.

Conversely, let $\mathcal{F}$ satisfy (1) and (2). For any $\mathbf{c} \in \Gamma$, let $F(\mathbf{c})$ be the unique minimal face in $\mathcal{F}$ such that relint $\mathbf{c} \subset$ relint $\pi(F(\mathbf{c}))$. Let $\psi(\mathbf{c}) \in \operatorname{relint} \pi^{\vee}(N(F(\mathbf{c}))) \subset(\operatorname{ker} \pi)^{*}$ such that $F(\mathbf{c})=F_{\mathbf{c}, \psi(\mathbf{c})}$. Define

$$
\tilde{\Psi}: \Gamma \rightarrow(\operatorname{ker} \pi)^{*}
$$

as

$$
\tilde{\Psi}: \mathbf{c} \rightarrow \psi(\mathbf{c}) .
$$

Now if $F(\mathbf{c})=F_{\mathbf{c}, \psi}$ and $\mathbf{c}^{\prime}<\mathbf{c}$, then it follows from the coherent case that $F_{\mathbf{c}^{\prime}, \psi}$ equals the intersection of $F(\mathbf{c})$ with the preimage of the minimal face of $\pi(F(\mathbf{c}))$ containing $c^{\prime}$. Condition (2) implies $F\left(\mathbf{c}^{\prime}\right)=F_{\mathbf{c}^{\prime}, \psi}$, and the claim follows.

The minimal elements of $\mathcal{T}$ tie to the following:

Definition 3.3. $\mathcal{F}(\tilde{\Psi})$ is said to be tight if

$$
\operatorname{dim} \pi(F)=\operatorname{dim} F
$$

for all $F \in \mathcal{F}(\tilde{\Psi})$. (That is, $F$ does not drop dimension under the projection.) 
Proposition 3.4. $\mathcal{F}(\tilde{\Psi})$ is minimal if and only if $\mathcal{F}(\tilde{\Psi})$ is tight.

Proof. This is Lemma 9.5 of [33] whose proof for the coherent case extends to the general case. The proof goes as follows. Assume that $F(\tilde{\Psi})$ is minimal but there exists a face $F_{0} \in F(\tilde{\Psi})$ such that the projection $F_{0} \rightarrow \pi\left(F_{0}\right)$ drops dimension. Now choose a generic $\varphi \in(\operatorname{ker} \pi)^{*}$ and substitute each face $F$ from $\mathcal{F}(\tilde{\Psi})$ by the coherent string given by $\varphi$ in the projection $F \rightarrow \pi(F)$. This will do nothing to the "tight faces" (those with $\operatorname{dim}(F)=\operatorname{dim}(\pi(F)))$ but will refine the non-tight ones (e.g., $\left.F_{0}\right)$ in a locally coherent way.

The converse is easy. We omit further details.

\section{Coherent Costrings}

Let $q \in Q$ be any interior point. The point $q$ selects the following collection of cones in $\Delta(P)$ :

$$
\Delta(q)=\left\{N\left(P, F_{q, \psi}\right) \mid \psi \in(\operatorname{ker} \pi)^{*}\right\} .
$$

The collection only depends on the cell $\mathbf{c}(q)$. That is,

Lemma 4.1. $\Delta(q)=\Delta\left(q^{\prime}\right)$ if and only if $\mathbf{c}(q)=\mathbf{c}\left(q^{\prime}\right)$.

Proof. $\Delta(q)=\Delta\left(q^{\prime}\right)$ if and only if $F_{q, \sigma}=F_{q^{\prime}, \sigma}$ for all $\sigma \in \Gamma^{*}$ if and only if $[q]_{\sigma}=\left[q^{\prime}\right]_{\sigma}$ for all $\sigma \in \Gamma^{*}$ if and only if $\mathbf{c}(q)=\mathbf{c}\left(q^{\prime}\right)$.

Thus we may denote $\Delta(q)$ by $\Delta(\mathbf{c})$ if $q \in \operatorname{relint} \mathbf{c}(\mathbf{c} \in \Gamma)$.

Definition 4.2. $\Delta(\mathbf{c})$ is called a coherent costring. Let $\mathcal{T}_{\mathrm{coh}}^{*}=\{\Delta(\mathbf{c}) \mid \mathbf{c} \in \Gamma\}$ be the set of all coherent costrings, partially ordered by

$$
\Delta(\mathbf{c}) \leq \Delta\left(\mathbf{c}^{\prime}\right) \quad \text { if } \quad \bigcup \Delta(\mathbf{c}) \subset \bigcup \Delta\left(\mathbf{c}^{\prime}\right) .
$$

Proposition 4.3. Given any $\mathbf{c} \in \Gamma$. The collection $\Delta(\mathbf{c})$ satisfies the following properties:

(1) $\left\{\pi^{\vee}\left(N\left(P, F_{\mathbf{c}, \psi}\right)\right) \mid \psi \in(\operatorname{ker} \pi)^{*}\right\}$ is a cone subdivision of $(\operatorname{ker} \pi)^{*}$ without repetitions.

(2) $\pi^{\vee}\left(N\left(P, F_{\mathbf{c}, \psi}\right)\right) \subset \pi^{\vee}\left(N\left(P, F_{\mathbf{c}^{\prime}, \psi}\right)\right)$ if and only if

$$
N\left(P, F_{\mathbf{c}, \psi}\right)=\left(\pi^{\vee}\right)^{-1}\left(\pi^{\vee}\left(N\left(P, F_{\mathbf{c}, \psi}\right)\right)\right) \cap \pi^{\vee}\left(N\left(P, F_{\mathbf{c}^{\prime}, \psi}\right)\right) .
$$

Moreover, $\pi^{\vee}(\Delta(q))$ equals the normal fan $\Delta\left(P_{q}\right)$ if $q \notin \partial Q$.

Proof. The proof of (1) and (2) is analogous to that of Proposition 2.2 and is a careful check using definitions. For the last statement, just observe that $\pi^{\vee}\left(N\left(P, F_{q, \psi}\right)\right)=$ $N\left(P_{q}, P_{q}^{\psi}\right)$. We omit further details. 
Remark 4.4. (1) Like in Remark 2.4, two different coherent costrings may induce an identical cone subdivision of $(\operatorname{ker} \pi)^{*}$.

(2) Every cone in $\Delta(\mathbf{c})$ is strongly convex if $\mathbf{c}$ is not contained in the boundary of $Q$.

Proposition 4.5. The poset $\mathcal{T}_{\text {coh }}^{*}$ of all coherent costrings and the poset $\Gamma$ of all cells are anti-isomorphic. That is, $\mathcal{T}_{\text {coh }}^{*}=\Gamma^{\mathrm{opp}}$ and $\Gamma=\left(\mathcal{T}_{\text {coh }}^{*}\right)^{\mathrm{opp}}$.

Proof. Treat $\Delta$ as a function from $\Gamma$ to $\mathcal{T}_{\text {coh }}^{*}$. This is then a bijection by Lemma 4.1. Assume that $\mathbf{c}^{\prime} \leq \mathbf{c}$ are two cells. Pick $q_{\mathbf{c}}^{\prime} \in$ relint $\mathbf{c}^{\prime}$ and $q_{\mathbf{c}} \in$ relint $\mathbf{c}$. We have that $F_{q_{\mathbf{c}}^{\prime}, \psi} \leq F_{q_{\mathbf{c}}, \psi}$ for all $\psi \in(\operatorname{ker} \pi)^{*}$. That is, $N\left(P, F_{q_{\mathbf{c}}^{\prime}, \psi}\right) \geq N\left(P, F_{q_{\mathbf{c}}, \psi}\right)$ for all $\psi$. By definition, we obtain $\Delta\left(\mathbf{c}^{\prime}\right) \geq \Delta(\mathbf{c})$. That is, $\Delta$ is an order-reversing bijection.

\section{Locally Coherent Costrings}

Locally coherent costrings are generalizations of (global) coherent costrings. Geometrically, they are $\pi^{\vee}$-liftings of conical subdivisions of $(\operatorname{ker} p)^{*}$.

Definition 5.1. Let $\Delta_{0}$ be an arbitrary fan in $V^{*}$. A subcollection $\Delta$ of cones in $\Delta_{0}$ is called a locally coherent costring of $\pi^{\vee}:\left|\Delta_{0}\right| \subset V^{*} \rightarrow \pi^{\vee}\left(\left|\Delta_{0}\right|\right) \subset(\operatorname{ker} \pi)^{*}$ if it satisfies:

(1) $\left\{\pi^{\vee}(\sigma) \mid \sigma \in \Delta\right\}$ form a cone subdivision of $\pi^{\vee}\left(\left|\Delta_{0}\right|\right)$ without repetition.

(2) $\pi^{\vee}(\sigma) \subset \pi^{\vee}\left(\sigma^{\prime}\right)$ if and only if $\sigma=\left(\pi^{\vee}\right)^{-1}\left(\pi^{\vee}(\sigma)\right) \cap \sigma^{\prime}$.

It follows from the definition that if $\sigma \neq \sigma^{\prime} \in \Delta$, then $\pi^{\vee}(\sigma)$ and $\pi^{\vee}\left(\sigma^{\prime}\right)$ must intersect along a proper face. In particular, $\pi^{\vee}(\Delta)=\left\{\pi^{\vee}(\sigma) \mid \sigma \in \Delta\right\}$ is a fan in $W$. (Warning: a fan in our sense may not be strongly convex.)

Figure 1 illustrates an example of a (locally) coherent costring $\Delta$ in $\mathbb{R}^{3}$ with the projection $\pi^{\vee}(\Delta)$ in $\mathbb{R}^{2}$. (See [29] for pictures of locally coherent strings.)

The most interesting case where rich structures and duality emerge is the case when $\Delta_{0}$ is the normal fan $\Delta(P)$ of the polytope $P$.

Definition 5.2. The set of locally coherent costrings of $\pi^{\vee}:|\Delta(P)| \rightarrow(\operatorname{ker} \pi)^{*}$ is denoted by $\mathcal{T}^{*}=\mathcal{T}^{*}(P, Q)$. This set is equipped with a natural partial order by inclusion. Note that $\Gamma^{*}$ is a common refinement of $\pi^{\vee}(\Delta)$ for all $\Delta \in \mathcal{T}^{*}$.

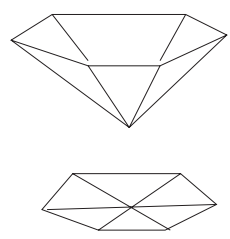

Fig. 1. A coherent costring with its projection. 
As before, we will actually construct all locally coherent costrings. So, again we consider a map

$$
\tilde{\Delta}: \Gamma^{*} \rightarrow Q
$$

satisfying the locally coherent condition: for any $\sigma>\sigma^{\prime}$,

$$
\text { relint }[\tilde{\Delta}(\sigma)]_{\sigma} \subset \operatorname{relint}\left[\tilde{\Delta}\left(\sigma^{\prime}\right)\right]_{\sigma^{\prime}} .
$$

$\tilde{\Delta}$ gives rise to a collection $\mathcal{C}(\tilde{\Delta})$ of cones in $\Delta(P)$ as follows:

$$
\mathcal{C}(\tilde{\Delta})=\left\{N\left(P, F_{\tilde{\Delta}(\sigma), \sigma}\right) \mid \sigma \in \Gamma^{*}\right\} .
$$

(Like the letter $\mathcal{F}$, in the rest of the paper, $\mathcal{C}$ denotes a collection of cones of a given fan.)

Theorem 5.3. Let $\tilde{\Delta}: \Gamma^{*} \rightarrow Q$ satisfy the locally coherent condition. $\mathcal{C}(\tilde{\Delta})$ is a locally coherent costring of $\pi^{\vee}:|\Delta(P)| \rightarrow(\operatorname{ker} \pi)^{*}$. Furthermore, every locally coherent costring of $\pi^{\vee}:|\Delta(P)| \rightarrow(\operatorname{ker} \pi)^{*}$ arises in this way.

Proof. The proof of this theorem is in a dual manner analogous to the case of locally coherent strings. As in the proof of Proposition 3.2, the argument can be reduced to the coherent case by using the same basic observation.

If the map $\tilde{\Delta}$ satisfies the local coherence condition and $\sigma^{\prime}<\sigma$, then $F_{\tilde{\Delta}(\sigma), \sigma}=F_{q, \sigma}$ and $F_{\tilde{\Delta}\left(\sigma^{\prime}\right), \sigma^{\prime}}=F_{q, \sigma^{\prime}}$ for some $q$ in relint $[\tilde{\Delta}(\sigma)]_{\sigma}$. So the claim follows from the coherent case.

Conversely, for any $\sigma \in \Gamma^{*}$, let $N(F(\sigma))$ be the minimal cone in $\mathcal{C}$ such that $\pi^{\vee}(N(F(\sigma)))$ contains $\sigma$. Choose $q(\sigma) \in$ relint $F(\sigma)$ to be a point such that $F(\sigma)=$ $F_{q(\sigma), \sigma}$. Define

$$
\tilde{\Delta}: \Gamma^{*} \rightarrow Q
$$

by

$$
\tilde{\Delta}: \sigma \rightarrow q(\sigma) .
$$

Now if $F(\sigma)=F_{\tilde{\Delta}(\sigma), \sigma}$ and $\sigma>\sigma^{\prime}$, then it follows from the coherent case that $N\left(F_{\tilde{\Delta}(\sigma), \sigma^{\prime}}\right)$ equals the intersection of $N\left(F_{\tilde{\Delta}(\sigma), \sigma}\right)$ with the preimage of the minimal face of $\pi^{\vee}(F(\sigma))$ containing $\sigma^{\prime}$. Condition (2) implies $N\left(F_{\tilde{\Delta}(\sigma), \sigma^{\prime}}\right)=N\left(F_{\tilde{\Delta}\left(\sigma^{\prime}\right), \sigma}\right)$ and the claim follows.

Note that the cone $N\left(P, F_{\tilde{\Delta}(\sigma), \sigma}\right)$ is strongly convex if $\tilde{\Delta}(\sigma)$ is not contained in the boundary of $Q$. Also, in view that $\Gamma^{*}=\mathcal{T}_{\text {coh }}$, every locally coherent costring $\mathcal{C}(\Delta)$ singles out a unique minimal element from any given coherent string $\mathcal{F}(\sigma) \in \mathcal{T}_{\text {coh }}$, i.e., the minimal face in $\mathcal{F}(\sigma)$ such that its image contains the cell $\tilde{\Delta}(\sigma)$.

Definition 5.4. A locally coherent costring $\mathcal{C}(\tilde{\Delta})$ is tight if $\operatorname{dim} \pi^{\vee}(\sigma)=\operatorname{dim} \sigma$ for every $\sigma \in \mathcal{C}(\tilde{\Delta})$. 
Analogous to Proposition 3.4 for locally coherent strings, we have the following proposition for locally coherent costrings whose proof is similar to that of Proposition 3.4 and is left to the reader.

Proposition 5.5. A locally coherent costring $\mathcal{C}(\tilde{\Delta})$ is tight if and only if it is minimal.

\section{Virtual Chambers}

Virtual cells are generalizations of the real cells. In toric geometry, real cells define projective quotient varieties, while virtual cells define (not necessarily projective) quotient varieties.

We refer to [13] for a treatment of virtual chambers in the case of triangulations of a point configuration.

Any map $\tilde{\mathbf{c}}: \Gamma^{*} \rightarrow Q$ gives rise to a collection of faces of $P$ as follows:

$$
\mathcal{F}(\tilde{\mathbf{c}})=\left\{F_{\tilde{\mathbf{c}}(\sigma), \sigma} \mid \sigma \in \Gamma^{*}\right\} .
$$

Definition 6.1. $\mathcal{F}(\tilde{\mathbf{c}})$ is called a virtual cell if $\tilde{\mathbf{c}}$ satisfies the following locally coherent condition: for $\sigma \geq \sigma^{\prime}$,

$$
\text { relint }[\tilde{\mathbf{c}}(\sigma)]_{\sigma} \subset \operatorname{relint}\left[\tilde{\mathbf{c}}\left(\sigma^{\prime}\right)\right]_{\sigma^{\prime}} .
$$

We use $\Gamma_{\text {vir }}=\Gamma_{\text {vir }}(P, Q)$ to denote the set of all virtual cells, partially ordered by inclusion: $\tilde{\mathbf{c}} \leq \tilde{\mathbf{c}}^{\prime}$ if $\bigcup \mathcal{F}(\tilde{\mathbf{c}}) \subset \bigcup \mathcal{F}\left(\tilde{\mathbf{c}}^{\prime}\right)$. Maximal virtual cells are called virtual chambers.

Theorem 6.2. Assume that a map $\tilde{\mathbf{c}}: \Gamma^{*} \rightarrow Q$ satisfies the locally coherent condition. Then the virtual cell $\mathcal{F}(\tilde{\mathbf{c}})$ satisfies the following property: for any coherent string $\Psi$, $\mathcal{C}(\tilde{\mathbf{c}}) \cap \mathcal{F}(\Psi)$ is non-empty and contains exactly one minimal element. Furthermore, every collection of the faces of $P$ satisfying the above property arises in this way.

Proof. Let $\mathcal{F}=\mathcal{F}(\sigma) \in \mathcal{T}_{\text {coh }}$ with $\sigma \in \Gamma^{*}$. Clearly, both $\mathcal{F}(\sigma)$ and $\mathcal{F}(\tilde{\mathbf{c}})$ contain the element $F_{\tilde{\mathbf{c}}(\sigma), \sigma}$. That is, the intersection is not empty. Now given any two faces $F_{p, \sigma}$ and $F_{q, \sigma} \in \mathcal{F}(\sigma) \cap \mathcal{F}(\tilde{\mathbf{c}})$, either they have a common face or they do not meet at all. If they do not meet at all, $\pi^{\vee}\left(N\left(F_{p, \sigma}\right)\right)$ and $\pi^{\vee}\left(N\left(F_{q, \sigma}\right)\right)$ will not have a nontrivial common face. Hence they meet in relative interiors (both contain $\sigma$ ). However, as members of the locally coherent costring $\mathcal{C}(\Delta)$ (transported from $\Delta=\tilde{c}$ ), they cannot meet in relative interiors. This shows that $F_{p, \sigma} \cap F_{q, \sigma}$ is a common face of each. Clearly, $\mathcal{F}(\sigma) \cap \mathcal{F}(\tilde{\mathbf{c}})$ must, as a subset of a coherent string $\mathcal{F}(\sigma)$ and with the property that every two members have a common face, have a unique minimal element.

On the other hand, given a collection $\mathcal{F}$ that meets every coherent string $\mathcal{F}(\sigma)$ in exactly one minimal element. Let $F(\sigma) \in \mathcal{F} \cap \mathcal{F}(\sigma)$ be this minimal element. Pick a point $q_{\sigma} \in$ relint $\pi(F(\sigma))$. We obtain a map

$$
\tilde{\mathbf{c}}: \Gamma^{*} \rightarrow Q
$$

by sending $\sigma$ to $q_{\sigma}$. We now check that this map satisfies the locally coherent condition. For any $\sigma^{\prime} \leq \sigma$, we have that the subdivision $\{\pi(F) \mid F \in \mathcal{F}(\sigma)\}$ refines the subdivision 
$\left\{\pi(F) \mid F \in \mathcal{F}\left(\sigma^{\prime}\right)\right\}$. It follows that relint $\pi(F(\sigma)) \subset$ relint $\pi\left(F\left(\sigma^{\prime}\right)\right)$ because either $F(\sigma)=F\left(\sigma^{\prime}\right)$ or $\pi(F(\sigma))$ divides $\pi(F(\sigma))$ in its relative interiors. This checks the locally coherent condition.

We do not know whether the following two are equivalent:

(1) For any coherent string $\Psi, \mathcal{F}(\tilde{\mathbf{c}}) \cap \mathcal{F}(\Psi)$ is non-empty and contains exactly one minimal element.

(2) For any locally coherent string $\tilde{\Psi}, \mathcal{F}(\tilde{\mathbf{c}}) \cap \mathcal{F}(\tilde{\Psi})$ is non-empty and contains exactly one minimal element.

This is proved in [13] for virtual chambers of point configurations in general position.

\section{Virtual Cones}

Virtual cones are simply dual versions of virtual cells.

An arbitrary map $\tilde{\sigma}: \Gamma \rightarrow(\operatorname{ker} \pi)^{*}$ defines a collection $\mathcal{C}(\tilde{\sigma})$ of cones of $\Delta(P)$ :

$$
\mathcal{C}(\tilde{\sigma})=\left\{N\left(F_{\mathbf{c}, \tilde{\sigma}(\mathbf{c})}, P\right) \mid \mathbf{c} \in \Gamma\right\} .
$$

Definition 7.1. $\mathcal{C}(\tilde{\sigma})$ of cones of $\Delta(P)$ is called a virtual cone if $\tilde{\sigma}$ satisfies the locally coherent condition. The set of all virtual cones is denoted by $\Gamma_{\mathrm{vir}}^{*}=\Gamma_{\mathrm{vir}}^{*}(P, Q)$, partially ordered by inclusion.

Theorem 7.2. Let $\tilde{\sigma}: \Gamma \rightarrow(\operatorname{ker} \pi)^{*}$ satisfy the locally coherent condition. Then the virtual cone $\mathcal{C}(\tilde{\sigma})$ satisfies the following property. For every coherent costring $\mathcal{C}(\mathbf{c})$, $\mathcal{C}(\tilde{\sigma}) \cap \mathcal{C}(\mathbf{c})$ is nonempty and contains exactly one maximal member. Furthermore, every collection of cones of $\Delta(P)$ that satisfies the above property arises in this way.

Proof. Transporting $\mathcal{C}(\mathbf{c})$ and $\mathcal{C}(\tilde{\sigma})$ to their corresponding face collections $\mathcal{F}(\mathbf{c})$ and $\mathcal{F}(\tilde{\Psi})$ where $\tilde{\Psi}=\tilde{\sigma}$, we see immediately that $\mathcal{F}(\tilde{\Psi}) \cap \mathcal{F}(\mathbf{c})$ contains a unique minimal element (i.e., the intersection of all the faces of $\mathcal{F}(\tilde{\Psi})$ that contain $\mathbf{c})$. Translating back, this means that $\mathcal{C}(\tilde{\sigma}) \cap \mathcal{C}(\mathbf{c})$ contains exactly one maximal member.

Again, the following two may be equivalent:

(1) $\mathcal{C}(\tilde{\sigma})$ meets every coherent costring in exactly one maximal member.

(2) $\mathcal{C}(\tilde{\sigma})$ meets every locally coherent costring in exactly one maximal member.

Before we move on, it may be worthwhile to summarize the dualities that we have so far

Theorem 7.3. The following always hold:

(1) A collection $\mathcal{F}$ of the faces of $P$ is a locally coherent string of $\pi: P \rightarrow Q$ if and only if $\mathcal{C}=\{N(P, F) \mid F \in \mathcal{F}\}$ is a virtual cone of $\Delta(P) \rightarrow(\operatorname{ker} \pi)^{*}$. 
(2) A collection $\mathcal{F}$ of the faces of $P$ is a virtual cell of $\pi: P \rightarrow Q$ if and only if $\mathcal{C}=\{N(P, F) \mid F \in \mathcal{F}\}$ is a locally coherent costring of $\Delta(P) \rightarrow(\operatorname{ker} \pi)^{*}$.

(3) A collection $\mathcal{C}$ of the cones of $\Delta(P)$ is a locally coherent costring of $\Delta(P) \rightarrow$ $(\operatorname{ker} \pi)^{*}$ if and only if $\mathcal{F}=\{F \mid N(P, F) \in \mathcal{C}\}$ is a virtual cell of $P \rightarrow Q$.

(4) A collection $\mathcal{C}$ of the cones of $\Delta(P)$ is a virtual cone of $\Delta(P) \rightarrow(\operatorname{ker} \pi)^{*}$ if and only if $\mathcal{F}=\{F \mid N(P, F) \in \mathcal{C}\}$ is a locally coherent string of $\pi: P \rightarrow Q$.

Furthermore, the correspondence in each of (1)-(4) reverses the inclusion-induced partial orders.

Proof. This theorem is a corollary to the combination of the results in the previous sections.

\section{Realizable Virtual Chambers}

In Section 6 a virtual cell was abstractly defined as a collection of faces arising in a certain locally coherent way. (Recall that it corresponds canonically to a locally coherent costring. See Theorem 7.3(2).) In this section we explain that many virtual cells can be obtained by topologically deforming the original polytopal cell complex $\Gamma$.

However, what deformation should be considered tamed (allowed) is quite subtle (thanks to the comment and suggestions (and patience) of one of the referees). The point is, if we start with a very special projection $\pi: P \rightarrow Q$, then a sufficiently general deformation of $\pi$ is likely to change the combinatorial structure drastically (e.g., to have undesirably more (virtual) chambers than the original complex has).

Let $P_{t}$ be a "sufficiently small" topological deformation of $P$. Equivalently, let $i: P \hookrightarrow V$ be the inclusion, we consider a small perturbation $i_{t}$ of $i$ and let $P_{t}=i_{t}(P)$.

If the projection $\pi: P \rightarrow Q$ is itself "sufficiently general", then any "sufficiently small" topological deformation is tamed (allowed). Here, "sufficiently general" means that every face $F$ of $P$ has $\operatorname{dim}(\pi(F))=\min \{\operatorname{dim}(Q), \operatorname{dim}(F)\}$. (In other words, that $\pi$ is bijective up to faces of $P$ of $\operatorname{dimension} \operatorname{dim}(Q)$.)

For an arbitrary projection $\pi: P \rightarrow Q$, a "sufficiently small" topological deformation is tamed (allowed) if the deformation preserves the face lattice of $\pi(F)$ for every face $F$ of $P$. (In particular, applied to the trivial face $P$ of $P$, this says that the deformation preserves the face lattice of $Q$.)

For a tamed deformation, if $F$ is a face of $P$, we write $F_{t}=i_{t}(F) . F_{t}$ is called a face of $P_{t}$. Let $Q_{t}=\pi\left(P_{t}\right)$. We call $\pi\left(F_{t}\right)$ a deformed subpolytope of $Q_{t}$.

Definition 8.1. The (deformed) cell in $Q_{t}$ containing a point $q \in Q_{t}$ is the intersection of $\pi$-images of all the faces of $P_{t}$ that contain the given point $q$. The set of all (deformed) cells is denoted by $\Gamma_{t}$, partially ordered by inclusion. Maximal cells are called chambers.

Figure 2 is a typical tamed deformation of a hexagon and its internal wall structures. Note that by the deformation, we gain one more (deformed) chamber, three more deformed one-dimensional cells and two more zero-dimensional cells (gain three but lose one). 

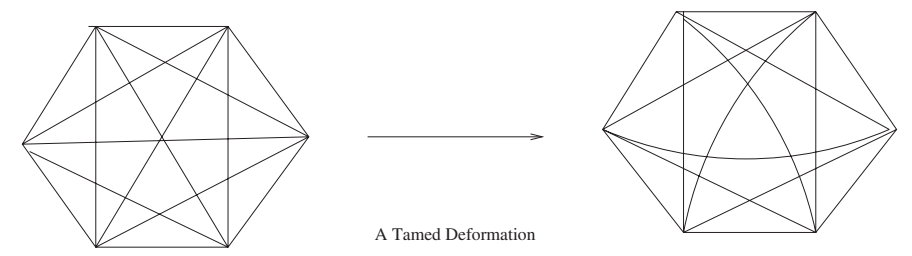

Fig. 2. A tamed deformation.

Figure 3 is not a tamed deformation because it "disconnects" the vertex in the upper right corner.

Let $T$ be a coherent string in $\mathcal{T}_{\text {coh }}=\mathcal{T}_{\text {coh }}(P, Q)$. Set

$$
T_{t}=\left\{F_{t} \mid F \in T\right\} .
$$

One can check that

\section{Lemma 8.2.}

(1) $\pi\left(T_{t}\right)$ forms a subdivision without repetition of $Q_{t}$ by deformed subpolytopes;

(2) $\Gamma_{t}$ is the common refinement of $\pi\left(T_{t}\right)$ for all $T \in \mathcal{T}_{\text {coh. }}$.

Proposition 8.3. A deformed cell in $\Gamma_{t}$ corresponds canonically to a virtual cell in $\Gamma$.

Proof. Each cell of $\Gamma_{t}$ specifies a unique minimal deformed subpolytope in $\pi\left(T_{t}\right)$ and thus a unique minimal face $F_{t}$ in $T_{t}$ for every $T \in T$. This leads to a unique minimal face $F$ in the original $T$. By Theorem 6.2, we conclude that every cell of $\Gamma_{t}$ gives rise to a virtual cell of $\pi: P \rightarrow Q$.

Definition 8.4. A virtual cell (chamber) that corresponds to some deformed cell (chamber) is called a realizable virtual cell (chamber). A deformed wall in $Q_{t}$ is called a virtual wall.

In Figure 2, the picture on the right, the central triangle is a typical realizable virtual chamber and the three curved walls are typical virtual walls.

Some very special realizable virtual chambers deserve special attention.
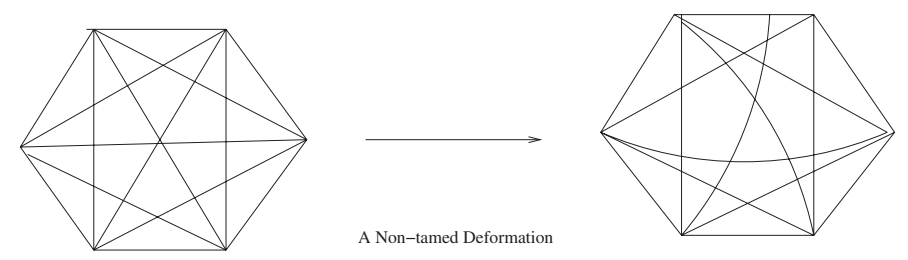

Fig. 3. A non-tamed deformation. 
Definition 8.5 [13]. We define lexicographic cells by induction. A vertex of $Q$ is lexicographic. Let $C \subset D$ be two faces of $Q$ with $\operatorname{dim} D=\operatorname{dim} C+1$ and let $\mathbf{c}$ be a lexicographic cell in $C$ of dimension $\operatorname{dim} C$. Then a cell in $D$ of $\operatorname{dimension} \operatorname{dim} D$ that has $\mathbf{c}$ as a face is lexicographic.

It is easy to see that the realizable virtual cell that corresponds to a lexicographic cell is independent of the deformation. From this, we get

Corollary 8.6. The subposet of realizable virtual cells is connected.

Proof. This is because every realizable virtual cell is connected to a lexicographic cell and lexicographic cells are invariant under a tamed deformation.

I learned from one of the referees that not all virtual chambers are realizable. They are if $\operatorname{dim} P-\operatorname{dim} Q \leq 3$ (see [3]). Also this section is related to the concept of "pseudochamber complex" in Section 4 of [3].

\section{Toric Varieties}

The rest of the paper deals with toric varieties. The basic references we use here are [16], [12] and [11].

Let $X^{d}$ be an arbitrary toric variety over the field of complex numbers, not necessarily quasi-projective, acted upon by the open dense torus $\left(\mathbb{C}^{*}\right)^{d}$. We study categorical (good) quotients of $X$ by a diagonalizable subgroup $G$ of $\left(\mathbb{C}^{*}\right)^{d}$. The method renders it useful in the combinatorial concepts of the previous sections.

Fix a lattice $M \cong \mathbb{Z}^{d}$ and its dual lattice $N$. A fan $\Delta_{0}$ in $N_{\mathbb{R}}=N \otimes \mathbb{R}$ consists of a finite collection of strongly convex rational polyhedral cones $\sigma \subset N_{\mathbb{R}}$ which are closed under intersection and taking faces.

Given (and fix) the fan $\Delta_{0}$ and a cone $\sigma$ in the fan, the dual cone

$$
\sigma^{\vee}=\left\{v \in M_{\mathbb{R}}:\langle v, \sigma\rangle \geq 0\right\}
$$

determines the semigroup algebra $\mathbb{C}\left[\sigma^{\vee} \cap M\right]$ which is finitely generated and the affine variety

$$
A_{\sigma}=\operatorname{Spec}\left(\mathbb{C}\left[\sigma^{\vee} \cap M\right]\right) .
$$

It turns out that these affine varieties can be glued together to get a toric variety

$$
X_{\Delta_{0}}=\bigcup_{\sigma \in \Delta_{0}} \operatorname{Spec}\left(\mathbb{C}\left[\sigma^{\vee} \cap M\right]\right) .
$$

Recall that $T_{N}=N \otimes \mathbb{C}^{*}=\operatorname{Hom}_{\mathbb{Z}}\left(M, \mathbb{C}^{*}\right)$ acts on $\mathbb{C}[M]$ by the formula

$$
\gamma_{n}(t) \chi^{m}=t^{\langle n, m\rangle} \chi^{m}
$$

where $\gamma_{n}$ is the one-parametric subgroup generated by $n \in N$.

$X_{\Delta_{0}}$ can also be constructed as a quotient of certain open subset of some affine space by a diagonalizable group. This is due to [11]. See also Section 12 . 


\section{Locally Coherent Costrings and Quotients}

Let $M^{2}$ be any sublattice of $M$. Consider the exact sequence

$$
0 \rightarrow M^{2} \rightarrow M \stackrel{\pi}{\rightarrow} M^{1} \rightarrow 0
$$

If we apply $\operatorname{Hom}_{\mathbb{Z}}\left(-, \mathbb{C}^{*}\right)$ to it, then we get the exact sequence

$$
1 \rightarrow \operatorname{Hom}_{\mathbb{Z}}\left(M^{1}, \mathbb{C}^{*}\right) \rightarrow \operatorname{Hom}_{\mathbb{Z}}\left(M, \mathbb{C}^{*}\right) \rightarrow \operatorname{Hom}_{\mathbb{Z}}\left(M^{2}, \mathbb{C}^{*}\right) \rightarrow 1 .
$$

The group $G=\operatorname{Hom}_{\mathbb{Z}}\left(M^{1}, \mathbb{C}^{*}\right)$ is the product of the torus $\operatorname{Hom}_{\mathbb{Z}}\left(M_{\text {free }}^{1}, \mathbb{C}^{*}\right)$ and the finite group $\operatorname{Hom}_{\mathbb{Z}}\left(M_{\mathrm{tor}}^{1}, \mathbb{C}^{*}\right)$. Let

$$
0 \rightarrow N^{1} \rightarrow N \stackrel{\pi^{\vee}}{\longrightarrow} N^{2}
$$

be the dual exact sequence where the image of $\pi^{\vee}$ has finite index in $N^{2}$. (We also use $\pi\left(\pi^{\vee}\right)$ to denote its $\mathbb{R}$-linear extension.)

We need a couple of lemmas to proceed.

Lemma 10.1. Let $\sigma_{0} \in \Delta_{0}$ be such that $\pi^{\vee}\left(\sigma_{0}\right)=\sigma$ is strongly convex. Then

(1) $\mathbb{C}\left[\sigma_{0}^{\vee} \cap M\right]^{G} \cong \mathbb{C}\left[\sigma^{\vee} \cap M^{2}\right]$;

(2) $A_{\sigma_{0}} / / G$ exists and is naturally identified with $A_{\sigma}$.

Proof. Assertion (2) obviously follows from (1). We only need to prove (1). On one hand, it follows from the definitions that $\mathbb{C}\left[\sigma^{\vee} \cap M^{2}\right] \subset \mathbb{C}\left[\sigma_{0}^{\vee} \cap M\right]^{G}$. On the other hand, any point of $\mathbb{C}[M]$ that is left invariant under the action of $G=\operatorname{Hom}_{\mathbb{Z}}\left(M^{1}, \mathbb{C}^{*}\right)$ must belong to $M_{2}$ (one can check this by the action formula given in Section 11). This leads to $\left[\sigma_{0}^{\vee} \cap M\right]^{G} \subset \sigma^{\vee} \cap M^{2}$. The lemma thus follows.

The lemma below gives a criterion for the separateness of a quotient.

Lemma 10.2. Let $\sigma$ and $\sigma^{\prime}$ be two cones in $\Delta_{0}$. The open subset $A_{\sigma} \cup A_{\sigma^{\prime}}$ has the separated categorical quotient $\left(A_{\sigma} \cup A_{\sigma^{\prime}}\right) / / G$ if and only if $\pi^{\vee}(\sigma) \cap \pi^{\vee}\left(\sigma^{\prime}\right)$ is a face of each.

Proof. By Lemma 10.1, the (possibly non-separated) quotient variety $A_{\sigma} \cup A_{\sigma^{\prime}} / / G$ is naturally identified with the variety $A_{\pi^{\vee}(\sigma)} \cup A_{\pi^{\vee}\left(\sigma^{\prime}\right)}$. By a standard fact from the theory of toric varieties (see Lemma 1.4 of [16]), this is separated if and only if $\pi^{\vee}(\sigma) \cap \pi^{\vee}\left(\sigma^{\prime}\right)$ is a common face of both.

Definition 10.3. A $G$-equivariant map $X \rightarrow Y$ is called a good quotient if $X$ admits a covering by invariant affine open subsets $U_{i}=\operatorname{Spec}\left(S_{i}\right)$ such that $Y=\bigcup_{i} U_{i} / / G$ where $U_{i} / / G=\operatorname{Spec}\left(S_{i}^{G}\right)$.

Definition 10.4. A locally coherent costring of $\pi^{\vee}: N \rightarrow N^{2}$ is called strongly convex if every cone $\pi^{\vee}(\sigma)(\sigma \in \Delta)$ is strongly convex. 
Recall that a locally coherent costring is tight (minimal) if the dimension of any cone in the locally coherent costring does not drop under the projection.

The main theorem of this section follows:

Theorem 10.5. Let $\Delta$ be a strongly convex locally coherent costring of $N \rightarrow N^{2}$. Then $U(\Delta)=\bigcup_{\sigma \in \Delta} A_{\sigma} \subset X$ is an invariant Zariski open subset such that:

(1) The separated categorical good quotient $U(\Delta) \rightarrow U(\Delta) / / G$ exists.

(2) $U(\Delta) / / G$ is the toric variety $X_{\pi^{\vee}(\Delta)}$ defined by the induced fan $\pi^{\vee}(\Delta)$.

(3) $U(\Delta) \rightarrow U(\Delta) / / G$ is a geometric quotient if and only if $\Delta$ is tight (minimal).

Proof. That $U(\Delta)$ is an invariant Zariski open subset is obvious.

(1) and (2) follow from the combination of Lemmas 10.1 and 10.2 and the construction of toric varieties. For much of it, it suffices to observe that $U(\Delta) / / G=\bigcup_{\sigma \in \Delta} A_{\sigma} / / G=$ $\bigcup_{\sigma \in \Delta} A_{\pi^{\vee}(\sigma)}$ (by Lemma 10.1(2)). Statement (3) is local. So to show it, it suffices to prove that $A_{\sigma} / / G$ is a geometric quotient for each $\sigma \in \Delta$. Since $A_{\sigma} / / G$ is a categorical quotient, it is geometric if for every $x \in A_{\sigma}, G_{x}$ is finite (hence all orbits are closed and of maximal dimension). Let $N_{\sigma}$ be the lattice generated by $\sigma$. Then one can check that $T_{N_{\sigma}}=N_{\sigma} \otimes \mathbb{C}^{*}$ is the isotropy subgroup of any point in the $T$-orbit $\mathcal{O}_{\sigma} \subset A_{\sigma}$ determined by $\sigma$. Moreover, the isotropy subgroup of any point in $A_{\sigma}$ is contained in $T_{N_{\sigma}}$. So a point in $A_{\sigma}$ has an isotropy subgroup in $G=T_{N^{1}}$ of positive dimension if and only if $\operatorname{dim} T_{N^{1}} \cap T_{N_{\sigma}}>0$ if and only if $N^{1} \cap N_{\sigma} \neq\{0\}$ if and only if $\sigma$ drops dimension under the projection from $N$ to $N^{2}$. This proves the theorem.

As an immediate corollary of Theorem 10.5(3), we have

Corollary 10.6. If $\Delta$ is tight, then $U(\Delta)$ is a toric variety which is a principal $G$ bundle over $U(\Delta) / / G$, assuming that $G$ acts on $U(\Delta)$ freely. Moreover, all principal $G$-bundles among toric varieties arise this way.

Remark 10.7. As in the very general case of quotient theory, degenerate quotients exist. These are the quotients whose dimensions are less than expected (that is, smaller than $\operatorname{dim} X-\operatorname{dim} G)$. In our toric situation, this will be the case if the induced fan $\pi^{\vee}(\Delta)$ is not strongly convex (that is, some projection $\pi^{\vee}(\sigma)$ contains a non-trivial vector space, or equivalently, $\sigma \cap N^{1}$ is infinite). In this case, $U(\Delta) / / G$ is the toric variety defined by a reduction $\operatorname{fan} \pi^{\vee}(\Delta)_{\text {red }}$ and is of dimension less than $\operatorname{dim} X-\operatorname{dim} G$.

\section{Virtual Chambers and Quotients}

Assume now that $X_{\Delta_{0}}$ is projective and equipped with a $T_{N}$-linearized ample line bundle $L$. This corresponds to a polytope $P$ in the dual lattice $M$ of $N$. Let $Q=\pi(P)$.

Corollary 11.1. Let $\tilde{\mathbf{c}}$ be a virtual (real) cell of the projection $\pi: P \rightarrow Q$ such that its corresponding locally coherent costring is strongly convex. Then it defines a quotient 
toric variety $X_{\tilde{\mathbf{c}}}$ whose defining fan is isomorphic to the fan in $(\operatorname{ker} \pi)^{*}$ induced by the images of the locally (global) coherent costring $\Delta(\tilde{\mathbf{c}})$.

Proof. This is a special case of Theorem 10.5.

It should be noted that in the case of real cells, the toric variety $X_{\tilde{\mathbf{c}}}$ has a standard construction (e.g., in [21], [10] or [19]): it is the projective toric variety given by the normal fan of the fiber $P \cap \pi^{-1}(q)$ where $q$ is any point in the relative interior of the real cell $\tilde{\mathbf{c}}$.

A strongly convex non-realizable virtual cell defines a non-projective quotient. Some strongly convex realizable virtual cells in fact define projective quotients. In Section 13 we show that the relation among quotients defined by realizable virtual cells shares the characteristics of the relation among projective quotients defined by real cells.

For theories on more general quotients, see [4], [22]-[24] and [26].

\section{Cox's Quotient Construction of Toric Varieties}

Let $\Delta$ be any fan in $N$ and let $X_{\Delta}$ be the corresponding toric variety. Cox has given a construction of $X_{\Delta}$ as the quotient of an open subset of $\mathbb{C}^{|\Delta(1)|}$ where $\Delta(1)$ is the set of one-dimensional cones of $\Delta[11]$. $\mathbb{C}^{|\Delta(1)|}$ comes equipped with a natural basis $\left\{e_{\rho}\right\}$ where $\rho$ is the first lattice point on an edge of $\Delta(1)$. There is canonical projection

$$
\pi^{\vee}: \mathbb{C}^{|\Delta(1)|} \rightarrow N \otimes \mathbb{R}
$$

defined by sending each $e_{\rho}$ to $\rho$. Let $G=\operatorname{Hom}_{\mathbb{Z}}\left(A_{d-1}\left(X_{\Delta}\right), \mathbb{C}^{*}\right)$ where $A_{d-1}\left(X_{\Delta}\right)$ is the Chow group of $(d-1)$-dimensional cycles in $X_{\Delta}$.

Corollary 12.1 [11]. Let the notations be as above.

(1) $\Delta$ canonically corresponds to a locally coherent costring of $\pi: \mathbb{C}^{|\Delta(1)|} \rightarrow N$ and the toric variety $X_{\Delta}$ is the quotient of $\mathbb{C}^{|\Delta(1)|}$ by the group $G$ defined by the above locally coherent costring.

(2) $X_{\Delta}$ is a geometric quotient if and only if $\Delta$ is simplicial.

Proof. (1) This is tautological. Given $\sigma \in \Delta$, let $\sigma$ (1) be the set of edges of $\sigma$. Clearly, $\left\{\operatorname{span}_{\rho \in \sigma(1)}\left\{e_{\rho}\right\} \mid \sigma \in \Delta\right\}$ is the desired locally coherent string.

(2) By Theorem 10.5(3), $X_{\Delta}$ is geometric if and only if $\Delta$ is tight. It is tight if and only if the projection $\operatorname{span}_{\rho \in \sigma(1)}\left\{e_{\rho}\right\} \rightarrow \operatorname{span}_{\rho \in \sigma(1)}\{\rho\}$ does not drop dimension. This can happen if and only if $\operatorname{span}_{\rho \in \sigma}\{\rho\}$ is itself simplicial (i.e., $\{\rho\}_{\rho \in \sigma}$ are linearly independent).

\section{Quotients Defined by Realizable Virtual Chambers}

Realizable virtual cells (i.e., deformed cells) are special. In this section we show that their corresponding quotients share some characteristics of projective quotients defined 
by real cells. We freely adopt the notations from Section 8 and also the terminology and results from [19].

Let $\mathbf{c}_{t}$ and $\mathbf{c}_{t}^{\prime}$ be two adjacent cells in $\Gamma_{t}$ such that the intersection

$$
\mathbf{c}_{t} \cap \mathbf{c}_{t}^{\prime}=\mathbf{c}_{t}^{0}
$$

is a facet of each. Then we have

$$
U\left(\mathbf{c}_{t}\right) \subset U\left(\mathbf{c}_{t}^{0}\right) \supset U\left(\mathbf{c}_{t}^{\prime}\right)
$$

and the inclusions induce a diagram of morphisms of algebraic varieties

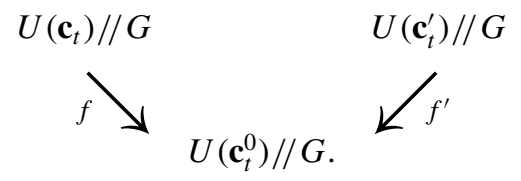

Set $\Sigma_{0}$ to be $U\left(\mathbf{c}_{t}^{0}\right) / / G \backslash U\left(\mathbf{c}_{t}^{0}\right)_{0} / / G$ where $U\left(\mathbf{c}_{t}^{0}\right)_{0}$ is the set of closed orbits of maximal dimension in $U\left(\mathbf{c}_{t}^{0}\right)$. Then $\Sigma_{0}$ admits a stratification by the so-called orbit types. Two points of $X$ have the same orbit type if their stabilizers in $G$ are identical. This induces a $G$-invariant stratification of $X$ which in turn induces a stratification of $\Sigma_{0}$.

As in the projective case (e.g., $t=0$ ), we have

Theorem 13.1. Assume that $\mathbf{c}_{t}^{0}$ is of codimension 1. Then

(i) $f$ and $f^{\prime}$ are isomorphisms over the complement to $\Sigma_{0}$;

(ii) over each connected component $\Sigma_{0}^{\prime}$ of a stratum of $\Sigma_{0}$, each fiber of $f\left(f^{\prime}\right)$ is isomorphic to a quotient of a weighted projective space of dimension $d\left(d^{\prime}\right)$ by the finite group $\pi_{0}\left(G_{z}\right)$ where $z$ is some point in $X^{\mathrm{ss}}\left(\mathbf{c}_{t}^{0}\right) \backslash X^{\mathrm{s}}\left(\mathbf{c}_{t}^{0}\right)$;

(iii) $d+d^{\prime}+1=\operatorname{codim} \Sigma_{0}^{\prime}$.

Proof. It is identical to the proof of Theorem 2.2 of [19].

Remark 13.2. For simplicity, we call the above birational transformation a Mori-type flip. Here we firmly give a few warnings about the use of the term Mori-type flips:

(1) Strictly, Mori flips are defined in the category of projective varieties. We borrow the term in the non-projective case as well.

(2) In a some special cases, some of our Mori-type flips may just correspond to blowups, while strictly Mori flips are birational transformations that are isomorphic in codimension 1 .

Proposition 13.3. Assume that $\mathbf{c}_{t}^{0}$ is of codimension 1 , lies on the boundary of $\Gamma_{t}$, and $\mathbf{c}_{t}$ is a chamber containing $\mathbf{c}_{t}^{0}$ as a face. Then the map $f: X^{\mathrm{ss}}\left(\mathbf{c}_{t}\right) / / G \rightarrow X^{\mathrm{ss}}\left(\mathbf{c}_{t}^{0}\right) / / G$ is fiber bundle whose typical fiber is isomorphic to a quotient of a weighted projective space by a finite abelian group.

Proof. It is identical to the proof of Theorem 2.1 of [19] (the finite abelian group action was unfortunately overlooked there). 
Corollary 13.4. Every lexicographic (virtual) cell corresponds to a projective quotient which is, modulo finite abelian group actions, a tower of weighted projective bundles over a fixed point variety.

Corollary 13.5. Every pair of quotient algebraic varieties defined by realizable virtual cells is connected by a sequence of Mori-type flips.

Proof. This is the geometric version of Corollary 8.6.

Assume that the two quotients are defined by a cell in $\Gamma_{t}$ and a cell $\Gamma_{s}$, respectively. Both quotients can be flipped to the same projective quotient defined by a lexicographic cell by crossing (virtual) walls.

\section{Some Notes Related to the Chow Quotient}

Besides invariant theoretic quotients, there is a canonical "quotient" space, the Chow quotient (see [21] and [20]). To recall the construction, note that the closure $\overline{G \cdot x}$ is a projective subvariety, and as $x$ ranges within a $G$-invariant open subset $U \subset X$ of generic points, these varieties will have the same dimension and degree. Let Chow $(X)$ be the Chow variety of all algebraic cycles in $X$. The assignment $G \cdot x \rightarrow \overline{G \cdot x}$ defines an embedding of $U / G$ into Chow $(X)$. The closure of the image of $U / G$ in Chow $(X)$ is the Chow quotient and is denoted by $X / /^{\mathrm{ch}} G$. The Chow quotient is a toric variety of the residue torus and its corresponding fan is the normal fan of the fiber polytope $\Sigma(P, Q)[21]$.

Assume now that we are in the situation as in Section 11. Then the $T_{N}$-linearized ample line bundle over $X$ determines a unique moment map $\mu_{T}$ for the $T_{N}$-action whose total image is the polytope $P$. It is a standard fact that the image of $\overline{\mathcal{O}}_{x}=\overline{T \cdot x}$ under $\mu_{T}$ is a face of $P$. Also, with respect to the same ample line bundle $L$, a moment map of the subtorus $G$-action is the composition

$$
\mu_{G}: X \rightarrow P \rightarrow Q
$$

where the last map is the projection we began with.

Let $C=\sum_{i} a_{i} C_{i} \in X / /^{\mathrm{ch}} G$ where $C_{i}=\overline{G \cdot x_{i}}$ for some $x_{i} \in X$. Then

Proposition 14.1. $\left\{\mu_{T}\left(\overline{\mathcal{O}}_{x_{i}}\right)\right\}_{i}$ is a coherent string of $P \rightarrow Q$. Moreover, every coherent string can be realized this way.

Proof. This is a reformulation of Proposition 3.6 of [21].

It is known that the Chow quotient maps to every projective GIT quotient. This fact extends to non-projective cases as well.

Corollary 14.2. Given any locally coherent costring $\Delta$, there is a natural projection from the Chow quotient $X / /^{\text {ch }} G$ to the quotient variety $U(\Delta) / / G$ that is a birational $T / G$-toric morphism. 
Proof. In the projective category, see the proofs in [20] and [21].

In our special situation the following proof is quick: just observe from the definitions that the fan of the Chow quotient refines the fan induced by any locally coherent costring. The birationality and the $T / G$-equivariancy are obvious.

\section{Bistellar Flips and Mori-Type Flips}

This section deals with the special case when $P$ is a simplex. Let $\mathcal{A}=\left\{a_{0}, \ldots, a_{n}\right\}$ be a finite lattice point set in $\mathbb{Z}^{d} \subset \mathbb{R}^{d}$ and $Q=\operatorname{conv}(\mathcal{A})$. We assume that the origin is an interior point of $Q$. We may consider $Q$ as the projection of the standard $n$-simplex $P$ by a suitable linear map $\pi$. In this case the minimal elements of $\left(\mathcal{T}_{\text {coh }}\right) \mathcal{T}$ are the (coherent) triangulations of $Q$ using solely the vertices from $\mathcal{A}$.

There is a vast literature on triangulations. See, for example, [13]-[15], [27], [30], [33] and the references therein.

Coherent triangulations correspond to the vertices of the secondary polytope $\Sigma(\mathcal{A})$ of $\mathcal{A}$. Two vertices are joined by an edge of $\Sigma(\mathcal{A})$ if and only if the corresponding triangulations are related by a bistellar flip. Here a bistellar flip is a local re-arrangement of a triangulation without introducing new vertices (see [33]).

Embed $\mathcal{A} \subset \mathbb{R}^{d} \subset \mathbb{R}^{d+1}$ in the affine hyperplane of height $1\left(x_{d+1}=1\right)$. Given any triangulation $T \in \mathcal{T}$, taking the cone over $T$, we get a fan $\Delta_{T}^{0}$ with the support cone $(\mathcal{A})$. To get a complete fan $\Delta_{T}$, we add the ray $R$ generated by the lattice point $0 \oplus(-1) \in \mathbb{R}^{d} \oplus \mathbb{R}$. The cones in $\Delta_{T} \backslash \Delta_{T}^{0}$ are generated by cones in $\partial(\operatorname{cone}(\mathcal{A}))$ and the ray $R$.

Proposition 15.1. $T$ is coherent if and only if $\Delta_{T}$ is projective.

Proof. The proof is almost tautological. Note that ( $T$ is coherent) $\Delta_{T}$ is projective if $(T)$ $\Delta_{T}$ possesses a strictly convex piecewise (affine) linear function. Thus if $\Delta_{T}$ possesses such a function, restricting it to $T$ will give a desired function for $T$. If $T$ has such a function, because $\Delta_{T}$ is obtained from the cone over $T$ be adding one edge $\mathbb{R}(0 \oplus(-1))$, this function can be extended to being a strictly convex piecewise linear function on $\Delta_{T}$ by assigning to $0 \oplus(-1)$ a generic and sufficiently large number (to assure that the extended linear functions are all different).

Example 15.2. Figure 4 is a non-coherent triangulation $T$ [33] and its corresponding non-projective fan $\Delta_{T}$. (see [16] for other examples of non-projective fans.)

Let $\mathcal{T}_{\text {coh }}^{0}$ be the subset of coherent triangulations that actually use all vertices of $\mathcal{A}$. Set

$$
G_{\hat{\mathcal{A}}}=\operatorname{Hom}_{\mathbb{Z}}\left(A_{d}\left(X_{\Delta_{T}}\right), \mathbb{C}^{*}\right)
$$

for any $T \in \mathcal{T}_{\text {coh }}^{0}$ where $A_{d}\left(X_{\Delta_{T}}\right)$ is the Chow group of $d$-dimensional cycles in $X_{\Delta_{T}}$. One checks that the group does not depend on the choice of $T$. The group $G_{\hat{\mathcal{A}}}$ acts on the complex vector space $(\mathbb{C})^{|\mathcal{A}|+1}$ via the inclusion $G_{\hat{\mathcal{A}}} \subset\left(\mathbb{C}^{*}\right)^{|\mathcal{A}|+1}$. 

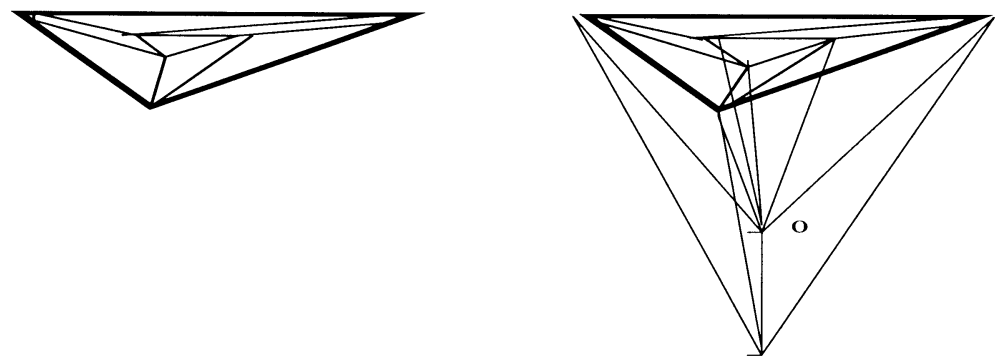

Fig. 4. Example 15.2.

Now by Proposition 15.1, the Cox Theorem (Corollary 12.1) and Theorem 13.1, we have

\section{Proposition 15.3.}

(1) There is canonical correspondence between the triangulations of $Q$ and quotient varieties of $\mathbb{C}^{|\mathcal{A}|+1}$ by the group $G_{\hat{\mathcal{A}}}$;

(2) under this correspondence, coherent triangulations correspond to projective quotient varieties, not necessarily coherent triangulations correspond to not necessarily projective quotient varieties;

(3) a bistellar flip between two triangulations transports to a Mori-type flip ${ }^{2}$ between their corresponding quotients, assuming in addition that the quotients are defined by realizable virtual chambers in the same deformed chamber complex.

\section{Acknowledgments}

My thanks go to Bernd Sturmfels for suggesting that I investigate the perfect correspondences that appeared in this paper. This paper grew out of our conversation at Utah Algebraic Geometry Conference in 1995 and my visit to Berkeley in 1996 and I thank him for his suggestions, comments and encouragement. I owe thanks to Victor Reiner for saving me from an embarrassment. I thank Michel Brion and Günter Ziegler for useful conversations or correspondence regarding the materials in this paper, and also Andrzej Bialynicki-Birula for sending his papers to me.

I thank all four referees for their encouragement, careful reading and very critical comments, suggestions and corrections. Their inputs substantially improved the paper and are deeply appreciated. The last section in the previous version on virtual oriented matroid (see [9] and [25]) is removed completely at the suggestion of one of the referees. Section 8 (used to be Section 9) received most complaints and is re-written.

\footnotetext{
${ }^{2}$ See Remark 13.2.
} 


\section{References}

1. A. A'Campo-Neuen, Quotients of Toric Varieties with Curve Lifting Property, preprint, math.AG/0003204.

2. A. A'Campo-Neuen, F. Berchtold and J. Hausen, Categorical Quotients of Simplicial Toric Varieties, preprint, math.AG/0001131.

3. M. Azaola and F. Santos, The graphs of triangulations of a point configuration with $d+4$ vertices is 3-connected, Discrete Comput. Geom. 23 (2000), 489-536.

4. A. Bialynicki-Birula and A. Sommese, Quotients by $\mathbb{C}^{*} \times \mathbb{C}^{*}$ actions, Trans. Amer. Math. Soc. 289 (1985), 519-543.

5. A. Bialynicki-Birula and J. Świecicka, Open subsets in projective spaces with a good quotient by an action of a reductive group, Transformation Groups 1(3) (1996), 153-186.

6. A. Bialynicki-Birula and J. Świecicka, A recipe for finding open subsets of vector spaces with a good quotient, preprint (1999).

7. L. J. Billera, M. Kapranov and B. Sturmfels, Cellular strings on polytopes, Proc. Amer. Math. Soc. 122 (1994), 549-555.

8. L. J. Billera and B. Sturmfels, Fiber polytopes, Ann. of Math. 135 (1992), 527-549.

9. A. Björner, M. Las Vergnas, B. Sturmfels, N. White and G. Gunter, Oriented Matroids, Cambridge University Press, Cambridge, 1992.

10. M. Brion and C. Procesi, Action d'un tore dans une variété projective, in A. Connes, M. Duflo, A. Joseph and R. Rentschler (eds.), Operator Algebras, Unitary Representations, Enveloping Algebras, and Invariant Theory, Progress in Mathematics, vol. 192, Birkhäuser, Basel, 1990, pp. 509-539.

11. D. Cox, The homogeneous coordinate ring of a toric variety, J. Algebra Geom. 4 (1995), 17-50.

12. D. Cox, Recent developments in toric geometry, Proc. Alg. Geom. Symposium, Santa Cruz, 1995, pp. 389-436. In J. Kollár, R. Lazarsfeld and D. Morrison (eds.), Algebraic Geometry.

13. J. de Loera, S. Hosten, F. Santos and B. Sturmfels, The polytope of all triangulations of a point configuration, Doc. Math. J. DMV 1 (1996), 103-119.

14. P. Edelman and V. Reiner, The higher Stasheff-Tamari posets, Mathematika 43 (1996), 127-154.

15. P. Edelman and V. Reiner, Visibility complexes and the Baues problem for triangulations in the plane, Discrete Comput. Geom. 20 (1998), 35-59.

16. W. Fulton, Introduction to Toric Varieties, Annals of Mathematical Studies, vol. 131, Princeton University Press, Princeton, NJ, 1993.

17. W. Fulton and B. Sturmfels, An intersection theory of toric varieties, Toplology 36(2) (1997), 335-353.

18. H. Hamm, Very good quotients of toric varieties, in J. W. Bruce et al. (eds.), Real and Complex Singularities (Proceedings of the 5th workshop, Sao Carlos, Brazil, July 27-31, 1998), Research Notes in Mathematics, vol. 412, Chapman \& Hall/CRC, Boca Raton, FL, 2000, pp. 61-75.

19. Y. Hu, The geometry and topology of quotient varieties of torus actions, Duke Math. J. 68 (1992), 151-183.

20. M. Kapranov, Chow quotients of Grassmannians I, Proc. I.M. Gelfand Seminar, American Mathematical Society, Providence, RI, 1993, pp. 29-110.

21. M. Kapranov, B. Sturmfels and A. Zalevenskii, Quotients of toric varieties, Math. Ann. 290 (1991), 643-655.

22. S. Keel and S. Mori, Quotients by groupoids, Ann. of Math. (2) 145(1) (1997), 193-213.

23. J. Kollár, Quotient spaces modulo algebraic groups, preprint (1995).

24. J. Kollár, Algberaic groups acting on schemes, a section of a book in preparation, private communication (1995).

25. R. MacPherson, Combinatorial differential manifolds, in Topology Methods in Modern Mathematics: A Symposium in Honor of John Milnor's Sixtieth Birthday, Stony Brook, NY, 1991, Publish or Perish, Houston, TX, 1993, pp. 203-211.

26. D. Mumford, J. Fogarty and F. Kirwan, Geometric Invariant Theory, Springer-Verlag, Berlin, 1994. (Referred to as GIT.)

27. T. Oda and H. Park, Linear Gale transforms and Gelfand-Kapranov-Zelevinsky decompositions, Tohoku Math J. 43 (1991), 375-399.

28. J. Rambau, Polyhedral subdivisions and projections of polytopes, Ph.D. Thesis, Fachbereich Mathematik, TU-Berlin, 1996.

29. J. Rambau and G. Ziegler, Projections of polytopes and the generalized Baues conjecture, Discrete Comput. Geom. 16(3) (1996), 215-237. 
30. V. Reiner, The generalized Baues problem, in L. Billera, A. Björner, C. Greene, R. Simion and R. Stanley (eds.), New Perspectives in Combinatorics, Cambridge University Press, Cambridge, 1999, pp. 293-336.

31. F. Santos, A point set whose space of triangulations is disconnected, J. Amer. Math. Soc. 13(3) (2000), 611-637.

32. J. Świecicka, Quotients of toric varieties by actions of subtori, Colloq. Math. 82(1) (1999), 105-116.

33. G. Ziegler, Lectures on Polytopes, Springer-Verlag, Berlin, 1994.

Received May 5, 2000, and in revised form February 2, 2001, November 26, 2001, and February 17, 2002.

Online publication July 24, 2002 